\title{
Meta-analysis and meta-modelling for diagnostic problems
}

\author{
Suphada Charoensawat ${ }^{1}$, Walailuck Böhning ${ }^{2}$, Dankmar Böhning ${ }^{3 *}$ and Heinz Holling ${ }^{2}$
}

\begin{abstract}
Background: A proportional hazards measure is suggested in the context of analyzing SROC curves that arise in the meta-analysis of diagnostic studies. The measure can be motivated as a special model: the Lehmann model for ROC curves. The Lehmann model involves study-specific sensitivities and specificities and a diagnostic accuracy parameter which connects the two.

Methods: A study-specific model is estimated for each study, and the resulting study-specific estimate of diagnostic accuracy is taken as an outcome measure for a mixed model with a random study effect and other study-level covariates as fixed effects. The variance component model becomes estimable by deriving within-study variances, depending on the outcome measure of choice. In contrast to existing approaches - usually of bivariate nature for the outcome measures - the suggested approach is univariate and, hence, allows easily the application of conventional mixed modelling.
\end{abstract}

Results: Some simple modifications in the SAS procedure proc mixed allow the fitting of mixed models for meta-analytic data from diagnostic studies. The methodology is illustrated with several meta-analytic diagnostic data sets, including a meta-analysis of the Mini-Mental State Examination as a diagnostic device for dementia and mild cognitive impairment.

Conclusions: The proposed methodology allows us to embed the meta-analysis of diagnostic studies into the well-developed area of mixed modelling. Different outcome measures, specifically from the perspective of whether a local or a global measure of diagnostic accuracy should be applied, are discussed as well. In particular, variation in cut-off value is discussed together with recommendations on choosing the best cut-off value. We also show how this problem can be addressed with the proposed methodology.

Keywords: Diagnostic accuracy, Mixed modelling, Random effects modelling, Cut-off value modelling, SROC modelling

\section{Background}

We are interested in the following setting occurring in the field of meta-analysis of diagnostic studies (Hasselblad and Hedges [1]; Sutton et al. [2]; Deeks [3]; Schulze et al. [4]): a variety of diagnostic studies are available providing estimates of the diagnostic measures of specificity $q=P(T=0 \mid D=0)$ as $\hat{q}_{i}=x_{i} / n_{i}$ and of sensitivity $p=P(T=1 \mid D=1)$ as $\hat{p}_{i}=y_{i} / m_{i}$, where $D=1$ and $D=0$ denote presence or absence of disease, respectively, and $T=1$ or $T=0$ denote positivity

\footnotetext{
${ }^{*}$ Correspondence: d.a.bohning@soton.ac.uk

3 Southampton Statistical Sciences Research Institute, Mathematics and Medical Statistics, University of Southampton, Southampton SO17 1BJ, UK
} Full list of author information is available at the end of the article or negativity of the diagnostic test, respectively, $x_{i}$ are the number of observed true-negatives out of $n_{i}$ healthy individuals, and $y_{i}$ are the number of observed truepositives out of $m_{i}$ diseased individuals, for $i=1, \ldots, k$, $k$ being the number of studies. For more details on the statistical modelling of the diagnostic data from a single study, see Pepe $[5,6]$. For a more detailed introduction to meta-analysis of diagnostic studies, see Holling et al. [7]. In the following, we will look at several examples mainly from medicine and psychology - for this special meta-analytic situation. In principle, however, applications could occur in all areas in which meta-analytic data is encountered; Swets [8] considers mainly psychological applications, but also mentions cases from engineering 
(quality control), manufacturing (failing parts in planes), metereology (correctness of weather predictions), information science (correctness of information retrieval), or criminology (correctness of lie detection test). We illustrate the special meta-analytic situation mentioned above with a meta-analysis on a diagnostic test on heart failure (see also Holling et al. [7]).

Example 1: Meta-Analysis of diagnostic accuracy of Brain Natriuretic Peptides (BNP) for heart failure. Doust et al. [9] provide a meta-analysis on the diagnostic accuracy of the brain natriuretic peptides (BNP) procedure as a diagnostic test for heart failure. According to the authors, diagnosis of heart failure is difficult, with both overdiagnosis and underdiagnosis occurring. The meta-analysis considers a range of diagnostic studies that use different reference standards (where a reference standard defines the presence or absence of disease). Here we only consider the eight studies (see Table 1) using the left ventricular ejection fraction of $40 \%$ or less as reference standard.

The cut-off value problem. A separate meta-analysis of sensitivity and specificity using the meta-analytic tools for independent binomial samples is problematic when the underlying diagnostic test utilizes a continuous or ordered categorical scale and different cut-off values have been used in different diagnostic studies. A simple variation of the cut-off value from study to study might lead to quite different values of sensitivity and specificity without any actual change in the diagnostic accuracy of the underlying test.

SROC curve. Due to this comparability problem for sensitivity and specificity, interest is usually focussed on the summary receiver operating characteristic (SROC) curve consisting of the pairs $(1-q(t), p(t))$ where $q(t)=$ $P(T<t \mid D=0)$ and $p(t)=P(T \geq t \mid D=1)$ for a continuous test $T$ with potential value $t$. For a given study $i$, $i=1, \cdots, k$, with potentially unknown cut-off value $t_{i}$,

\begin{tabular}{|c|c|c|c|c|c|}
\hline \multirow[b]{2}{*}{ Study $i$} & \multicolumn{2}{|c|}{ Diseased } & \multicolumn{2}{|c|}{ Healthy } & \multirow[b]{2}{*}{$n_{i}+m_{i}$} \\
\hline & $y_{i}(\mathrm{TP})$ & $m_{i}-y_{i}(\mathrm{FN})$ & $x_{i}(\mathrm{TN})$ & $n_{i}-x_{i}(\mathrm{FP})$ & \\
\hline Bettencourt 2000 & 29 & 7 & 46 & 19 & 101 \\
\hline Choy 1994 & 34 & 6 & 22 & 13 & 75 \\
\hline Valli 2001 & 49 & 9 & 78 & 17 & 153 \\
\hline Vasan 2002a & 4 & 6 & 1612 & 85 & 1707 \\
\hline Vasan 2002b & 20 & 40 & 1339 & 71 & 1470 \\
\hline Hutcheon 2002 & 29 & 2 & 102 & 166 & 299 \\
\hline Landray 2000 & 26 & 14 & 75 & 11 & 126 \\
\hline Smith 2000 & 11 & 1 & 93 & 50 & 155 \\
\hline
\end{tabular}

the pairs $\left(1-q\left(t_{i}\right), p\left(t_{i}\right)\right)$ can be estimated by $\left(1-\hat{q}_{i}, \hat{p}_{i}\right)=$ $\left(1-x_{i} / n_{i}, y_{i} / m_{i}\right)$ for $i=1, \ldots, k$. The SROC curve accommodates the cut-off value problem. Different pairs could have quite different values of specificity and sensitivity, but still reflect identical diagnostic accuracy. The SROC diagram for the meta-analysis on BNP and heart failure is given in Figure 1.

Clearly, there is a wide range of values for specificity and sensitivity. Nevertheless, as Figure 1 shows, the possibility that the pairs might stem from a common SROC curve (as given by the dashed curve in Figure 1) cannot be discarded. Since the SROC approach accommodates the cut-off value problem, it is commonly preferred to summary measures like the Youden index [10] or the diagnostic odds ratio [11]. In the following, we focus our analysis on the SROC curve.

Background of SROC modelling. SROC modelling has received considerable attention in the field and experienced several developments. An early model was suggested by Littenberg and Moses [12], [13] and has been used in practice frequently; Deeks [3] discusses its prominent role in modeling meta-analytic diagnostic study accuracy. Littenberg and Moses [13] suggest fitting $D=$ $\alpha+\beta S$, where $D=\log D O R=\log \frac{p}{1-p}-\log \frac{1-q}{q}$ is the $\log$-diagnostic odds ratio and $S=\log \frac{p}{1-p}+\log \frac{1-q}{q}$ is a measure for a potential threshold effect. After $\alpha$ and $\beta$ have been estimated from the data, the SROC-curve $(p$ vs. $1-q$ ) is reconstructed from the estimated values of $\alpha$ and $\beta$. The parameter $\alpha$ is interpreted as the summary

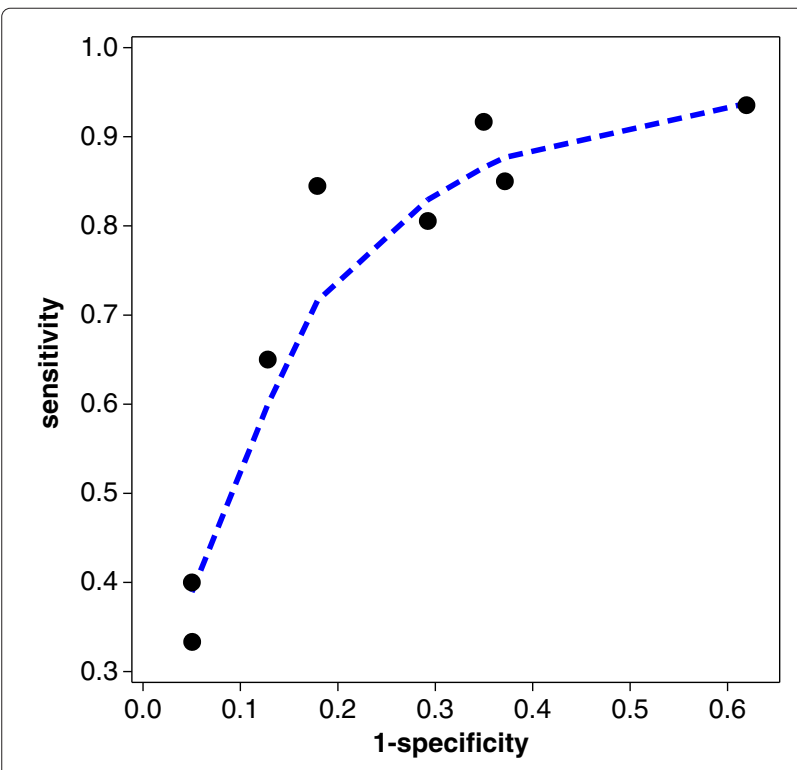

Figure 1 SROC diagram for BNP and heart failure: circles are the observed pairs of false positive rate and sensitivity, dashed curve is lowess smoother. 
$\log -D O R$, which is adjusted by means of $S$ for potential cut-off value effect.

A two-level approach has been suggested by Rutter and Gatsonis [14], which is typically given in the following notational form (Walter and Macaskill [15]): let $Z_{i j} \sim$ $\operatorname{Bi}\left(n_{i j}, \pi_{i j}\right)$, where $Z_{i j}$ is the number of test-positives in study $i$ for $\operatorname{arm} j(j=1$ is diseased, $j=2$ is non-diseased), $n_{i j}$ is the size of arm $j$ in study $i$ and $\pi_{i 1}$ is the sensitivity, $\pi_{i 2}$ is the false positive rate; the model is $\log \frac{\pi_{i j}}{1-\pi_{i j}}=$ $\left(\theta_{i}+\alpha_{i} D S_{i j}\right) \exp \left(-\beta D S_{i j}\right)$, where $\theta_{i}$ is an implicit threshold parameter for study $i, \alpha_{i}$ is the diagnostic accuracy parameter in study $i$, and $D S_{i j}$ represents a binary variable for the disease status. The parameter $\beta$ allows for an association between test accuracy and test threshold. When $\beta=0, \alpha_{i}$ is estimated by $D_{i}$ and $\theta_{i}$ is estimated by $S_{i} / 2$, where $D_{i}$ and $S_{i}$ are as for the Littenberg-Moses model. Furthermore, to account for between-study variation, a random effect is assumed for $\theta_{i} \sim N\left(\Theta, \tau_{\theta}^{2}\right)$ and $\alpha_{i} \sim N\left(\Lambda, \tau_{\alpha}^{2}\right)$, with $\theta_{i}$ and $\alpha_{i}$ being independent. As an alternative, a bivariate normal random-effects meta-analysis has been suggested by van Houwelingen et al. [16]; see also Reitsma et al. [17] and Arends et al. [18]. Harbord et al. [19] show that these models are closely related.

Paper overview. In the following, we propose a specific model, called the Lehmann model, which we believe is very attractive for the analysis of SROC curves. The model involves study-specific sensitivities and specificities and a diagnostic accuracy parameter which connects the two. The Lehmann model achieves flexibility by allowing the diagnostic accuracy parameter to become a random effect. In this it is similar to the Rutter-Gatsonis model, but differs in that it retains univariate dimensionality in its outcome measure and, hence, allows a mixed model approach in a more conventional way. In section "The proportional hazards measure", the proportional hazards measure is motivated as a specific form of SROC curve modelling and is compared to other approaches. Section "A mixed model approach" introduces the specific mixed model in which the log proportional hazards measure forms the outcome measure, the study factor is a normally distributed random effect (to cope with unobserved heterogeneity), and other observed covariates (such as gold standard or diagnostic test variation) are considered as fixed effects in the mixed model. Section "Results" considers various applications including a meta-analysis of the Mini-Mental State Examination to diagnose dementia or mild cognitive impairment. It also provides SAS-code for a simple execution of the suggested approach. In section "Discussion", the choice of outcome is discussed and the difference between global and local diagnostic accuracy measures highlighted. This is particularly of interest if observed cut-off value variation occurs in the meta-analysis and needs to be assessed.
Here a local criterion of diagnostic accuracy appears more appropriate. The paper ends with some brief conclusions and discussion in section "Conclusions".

\section{Methods}

\section{The proportional hazards measure}

Numerous summary measures for a pair of specificity and sensitivity have been suggested: we mention here the Youden index, $J_{i}=p_{i}+q_{i}-1$ [10], and the squared Euclidean distance to the upper left corner in the SROC diagram, $E_{i}=\left(1-p_{i}\right)^{2}+\left(1-q_{i}\right)^{2}$. [A review of summary measures is given in Liu [20].] Using an average over any of these measures might be problematic: not only might sensitivities and specificities be heterogeneous, this might also be true for the associated summary measures such as the Youden index or the Euclidean distance (as demonstrated by Figure 2 using the data of the meta-analysis of BNP and heart failure).

We suggest using the measure $\theta=\frac{\log p}{\log (1-q)}$, which relates the log-sensitivity to the log-false positive rate; we call it the proportional hazards (PH) measure. In Figure 3 we see that this measure shows a reduced variability for the meta-analysis of BNP and heart failure, making it more suitable as an overall measure in the meta-analysis of diagnostic studies or diagnostic problems. While the measure appears to be like any other summary measure of the pair sensitivity and specificity, it has a specific SROC-modelling background and motivation. We have mentioned previously the cut-off value problem: observed heterogeneity might be induced by cut-off value variation which could lead to different sensitivities and specificities - despite the accuracy of the diagnostic test itself not having changed - and might also lead to an induced heterogeneity in the summary measure. Hence, it is unclear whether the observed heterogeneity is due to heterogeneity in the diagnostic accuracy (authentic heterogeneity) or whether it has occurred due to cut-off value variation (artificial heterogeneity). This second form of heterogeneity can also occur when the background population changes with the study.

One of the features of the SROC approach is that it incorporates the cut-off value variation in a natural way; hence a measure modelling an ROC curve is favorable. We suggest the PH measure based upon the Lehman family in the following way:

$$
p=(1-q)^{\theta}
$$

This model was suggested by Le [21] for the ROC curve. It is an appropriate model since, for feasible $q,(1-q)^{\theta}$ is also feasible as long as $\theta$ is positive. Note that (1) is defined for all values of $p \in[0,1]$ and $q \in[0,1]$ whereas $\theta=\frac{\log p}{\log (1-q)}$ is only defined for $p \in(0,1)$ and $q \in(0,1)$. 


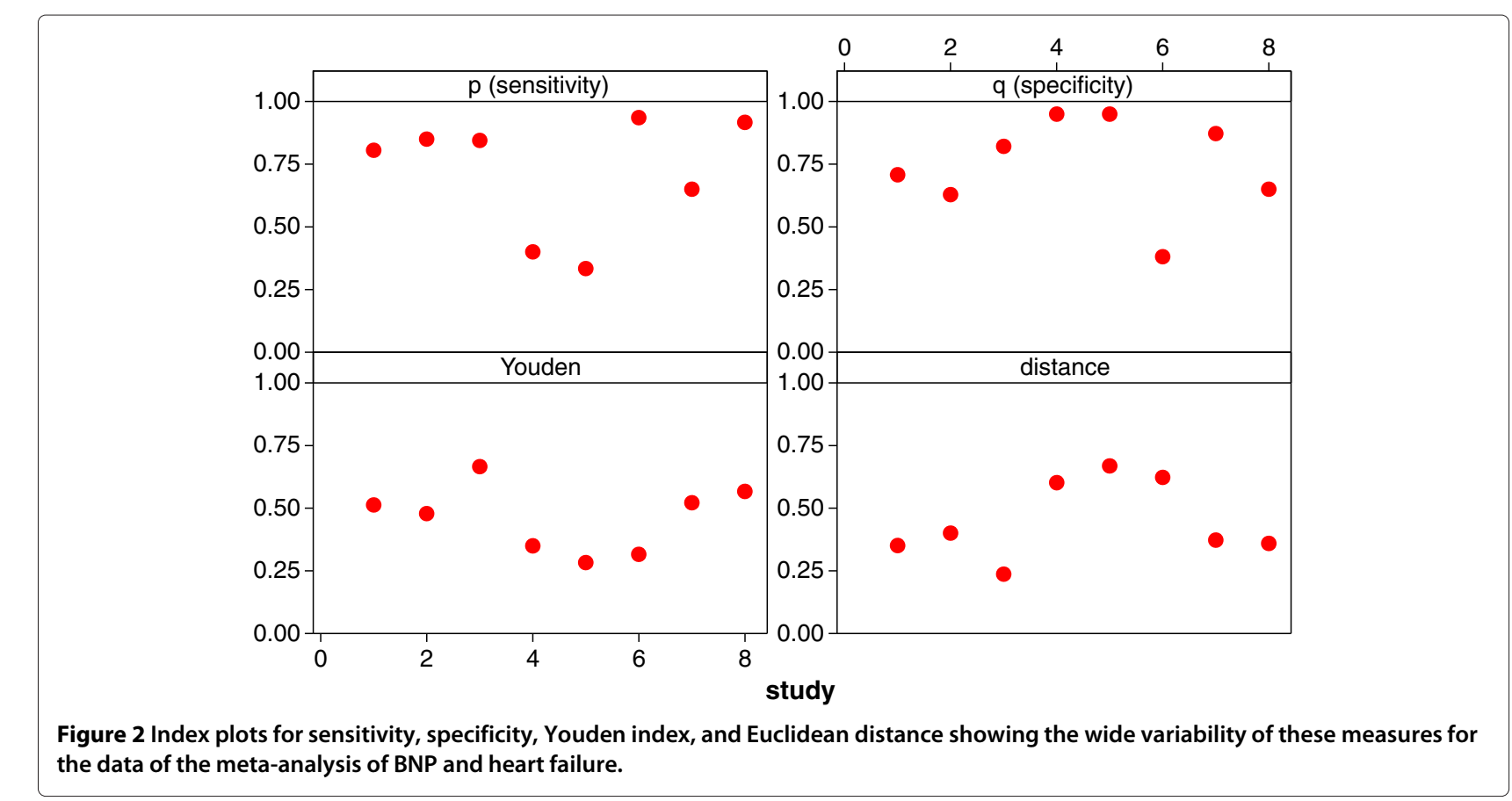

Population values of sensitivity and specificity of 1 are rarely realistic, although observed values of 1 for sensitivity and specificity do occur in samples. This can be coped with by using an appropriate smoothing constant such as estimating specificity as $\left(n_{i}-1\right) / n_{i}$ when $x_{i}=n_{i}$ and sensitivity as $\left(m_{i}-1\right) / m_{i}$ if $y_{i}=m_{i}$.

In Figure 4 we see a number of examples of the proportional hazards family. It becomes clear now why $\theta$ is called the proportional hazards measure. By taking logarithms on both sides of (1) we achieve

$$
\theta=\log p(t) / \log [1-q(t)]
$$

meaning if model (1) holds, the ratio of log-sensitivity to log-false positive rate is constant across the range of possible cut-off value choices $t$. Hence the name proportional hazards model, which was suggested in a paper by Le [21] and used again in Gönen and Heller [22]. The idea of representing an entire ROC curve in a single measure is illustrated in Figure 5. While sensitivity and specificity vary over the entire interval $(0,1)$, the value of $\theta$

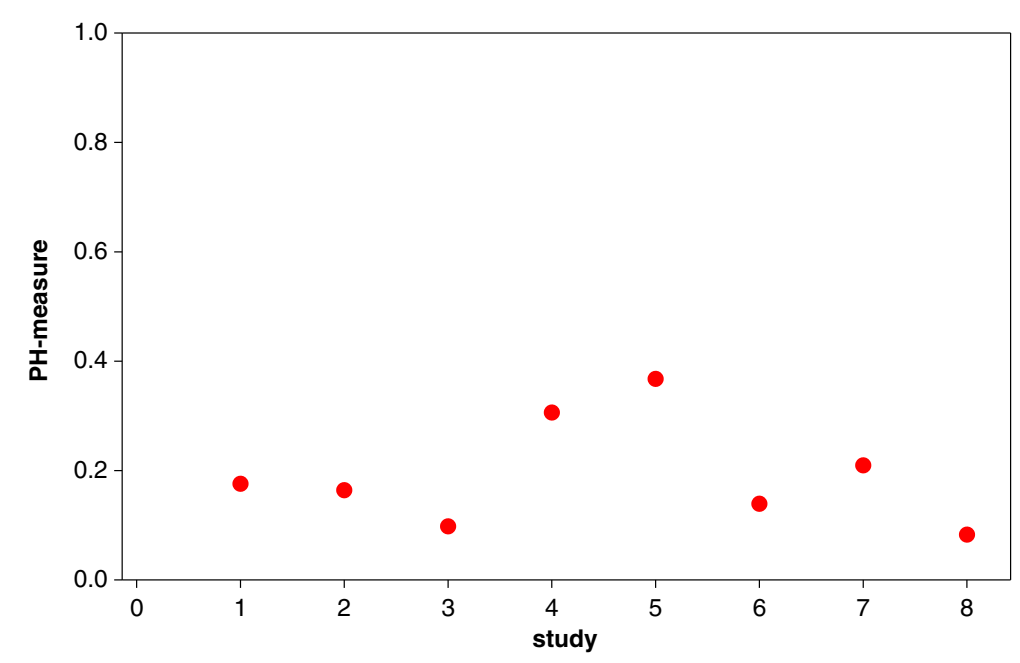

Figure 3 Index plots for the PH measures for the data of the meta-analysis of BNP and heart failure. 


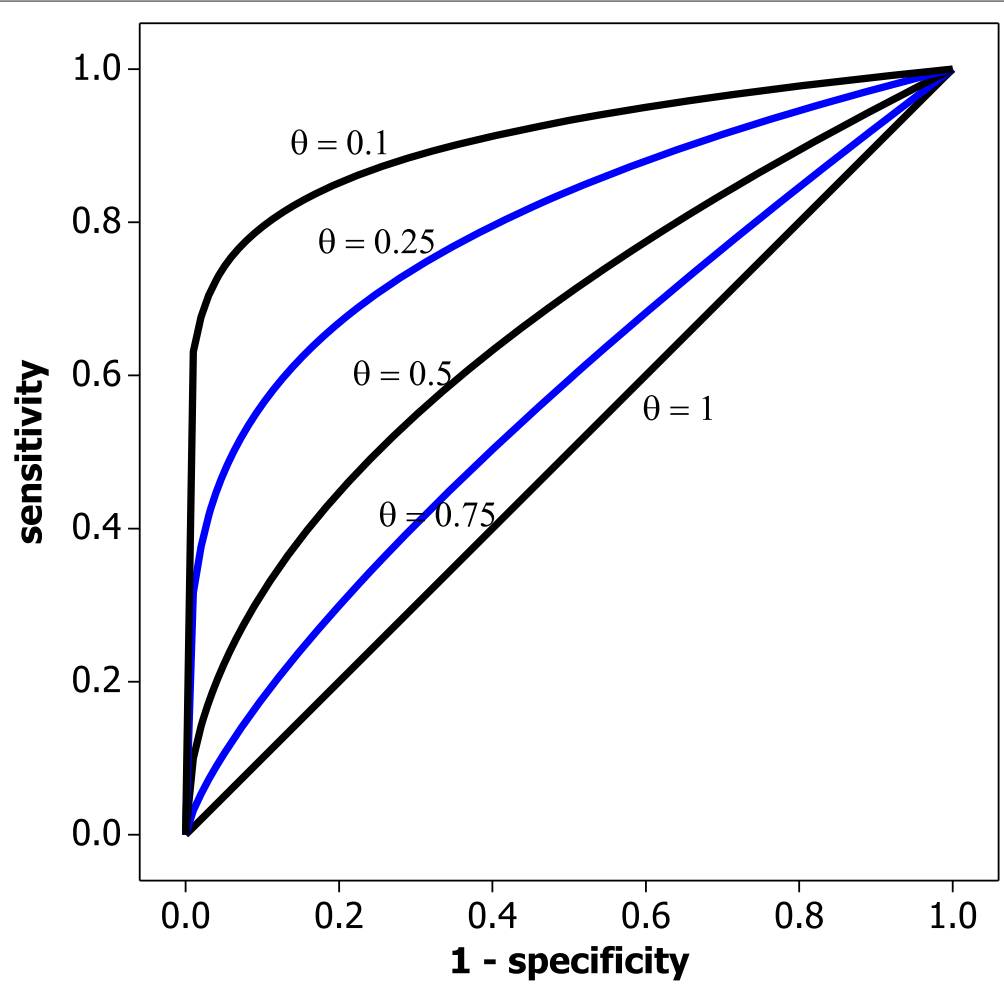

Figure 4 Some examples of the proportional hazards model for various values of $\theta$.

remains constant. Hence, log-sensitivity is proportional to the log-false positive rate. This assumption is similar to an assumption used for a model in survival analysis, where it is assumed that the hazard rate of interest is proportional to the baseline hazard rate; this might have motivated the choice of name used by Le [21] and Gönen and Heller [22] in this context.

However, it is not our intention to make the assumption that an entire SROC curve can be represented by model (1); the explanations above are instead meant as a
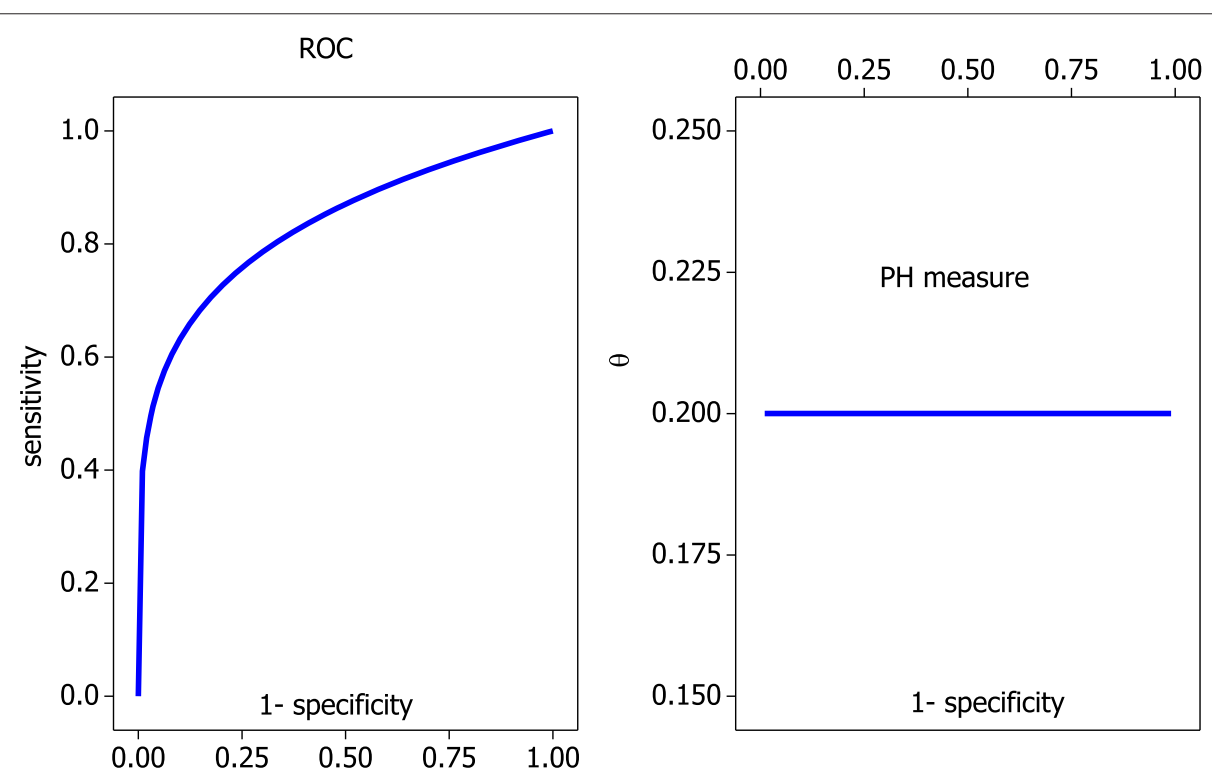

Figure 5 Proportional hazards model and associated PH measure. 
motivation that the PH-measure is not just another summary measure, but can be derived from a ROC modelling perspective. We envisage that each study, with associated pair of sensitivity and specificity, can be represented by a specific PH-model, as illustrated in Figure 6.

We see indeed that each pair of sensitivity and specificity can be associated with its own ROC curve provided by

$$
p=(1-q)^{\hat{\theta}_{i}}
$$

where $\hat{\theta}_{i}=\log \hat{p}_{i} / \log \left[1-\hat{q}_{i}\right]$, so that the curve (3) passes exactly through the point $\left(1-\hat{q}_{i}, \hat{p}_{i}\right)$.

Comparison to other approaches. It remains to be seen how appropriate the suggested proportional hazards model is and how it compares to other existing approaches. We emphasize that in our situation we have assumed that there is only one pair of sensitivity and false positive rate $\left(\hat{p}_{i}, 1-\hat{q}_{i}\right)$ per study $i$. Situations where several pairs per study are observed (such as in Aertgeerts et al. [23]) are rare. Hence, on the log-scale for sensitivity and false-positive rate, we are not able to identify any straight line model within a study with more than one parameter, since this would require at least two pairs of sensitivity and specificity per study; see also Rücker and Schumacher [24,25]. However, any one-parameter straight line model, such as the proposed proportional hazards model, is estimable within each study, although within-model diagnostics is limited since we are fitting the full within study model. Given that sample sizes within each diagnostic study are typically at least moderately large it seems reasonable to assume a bivariate normal distribution for $\log \hat{p}$ and $\log (1-\hat{q})$ with means $\log p$ and $\log (1-q)$ as well as variances $\sigma_{p}^{2}$ and $\sigma_{q}^{2}$, respectively, and covariance $\sigma$ with correlation $\rho=\sigma /\left(\sigma_{p} \sigma_{q}\right)$. This is very similar to the assumptions in the approach taken by Reitsma et al. [17] (see also Harbord et al. [19]), with the difference that we are using the log-transformation whereas in Reitsma et al. [17] logit-transformations are applied. Then, it is a well-known result that the mean of the random variable $\log \hat{p}$ (having unconditional mean $\log p$ ) conditional upon the value of the random variable $\log (1-\hat{q})$ (having unconditional mean $\log (1-q))$ is provided as

$E(\log \hat{p} \mid \log (1-\hat{q}))=\log p+\rho \frac{\sigma_{p}}{\sigma_{q}}[\log (1-\hat{q})-\log (1-q)]$,

which can be written as $\alpha+\theta[\log (1-\hat{q})]$ where $\alpha=$ $\log (p)-\theta \log (1-q)$ and $\theta=\rho \frac{\sigma_{p}}{\sigma_{q}}$. This is an important result since it means that, in the log-space, sensitivity and false-positive rate are linearly related. Furthermore, if $\alpha$ is zero, the proportional hazards model arises.

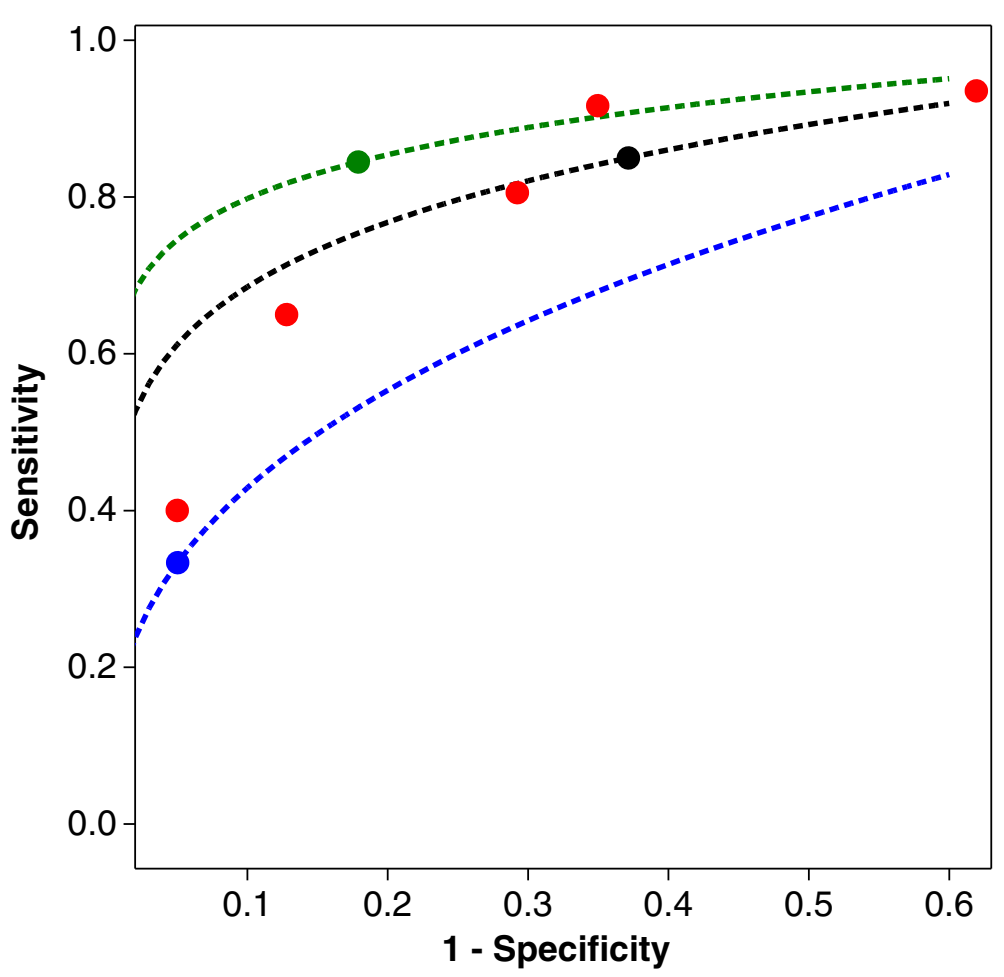

Figure 6 Meta-analysis of BNP and heart failure: each study is represented by its own PH model (1) - illustrated for 3 studies. 
The question then arises why not work with a straight line model

$$
\log p_{\mid \log (1-q)}=\alpha+\theta \log (1-q) .
$$

The answer is that such a model is not identifiable since we have only one pair of sensitivity and specificity observed in each study and it is not possible to uniquely determine a straight line by just one pair of observations since there are infinitely many possible lines passing through a given point in the $\log p-\log (1-q)$ space. However, the proportional hazards model as a slope-only model is identifiable and it is more plausible than other identifiable models such as the intercept-only model. Clearly, a logistic-transformation would be more consistent with the existing literature $[14,15]$ than the log-transformation. However, both models would give a perfect fit (within each study) since there are no degrees of freedom left for testing the model fit. The situation changes when there are repeated observations of sensitivity and specificity per study available. However, these meta-analyses with repeated observations of sensitivity and specificity according to cut-off value variation are extremely rare.

\section{A mixed model approach}

With the motivation of the previous sections in mind, we assume that $k$ diagnostic studies are available with diagnostic accuracies $\hat{\theta}_{1}, \cdots, \hat{\theta}_{k}$ where

$$
\hat{\theta}_{i}=\frac{\log \hat{p}_{i}}{\log \left(1-\hat{q}_{i}\right)}
$$

We assume the following linear mixed model for $\log \hat{\theta}_{i}$ :

$$
\log \hat{\theta}_{i}=\boldsymbol{\beta}^{\boldsymbol{T}} \boldsymbol{x}_{i}+\delta_{i}+\epsilon_{i}
$$

where $\mathbf{x}_{i}$ is a known covariate vector in study $i, \delta_{i}$ is a normally distributed random effect $\delta_{i} \sim N\left(0, \tau^{2}\right)$ with $\tau^{2}$ being an unknown variance parameter, and $\epsilon_{i} \sim N\left(0, \sigma_{i}^{2}\right)$ is a normally distributed random error with variance $\sigma_{i}^{2}$ known from the $i$-th study.

There are several noteworthy points about the mixed model (7). The response is measured on the log-scale, where the transformation improves the normal approximation and also brings the diagnostic accuracy into a wellknown link function family: the complementary log-log function. The difference of the probability for a positive test in the groups with and without the condition is measured on the complementary log-log scale. The fixed effect part involves a covariate vector $\mathbf{x}$ which could contain information on study level such as gold standard variation, diagnostic test variation, or sample size information. It should be noted that there are two variance components, $\tau^{2}$ and $\sigma_{i}^{2}$. It is important to have information on the second variance component. If the second component is unknown, even under the assumption of homogeneity $\sigma_{1}^{2}=\cdots=\sigma_{k}^{2}$, the variance component model would not be identifiable. Hence, we need to devote some effort to derive expressions for the within study variances; this can be accomplished using the $\delta$-method as discussed in the next section.

Within study variance. Let us consider (ignoring the study index $i$ for the sake of simplicity)

$$
\log \hat{\theta}=\log (-\log \hat{p})-\log [-\log (1-\hat{q})]
$$

and apply the $\delta$-method. Recall that the variance Var $T(X)$ of a transformed random variable $T(X)$ can be approximated as $\left[T^{\prime}(E(X))\right]^{2} \operatorname{Var}(X)$ assuming that the variance $\operatorname{Var}(X)$ of $X$ is known. Applying this $\delta$-method twice gives

$$
\operatorname{Var} \log (-\log \hat{p}) \approx \frac{\hat{p}(1-\hat{p}) / m}{\hat{p}^{2}(\log \hat{p})^{2}}
$$

and

$$
\operatorname{Var} \log (-\log (1-\hat{q})) \approx \frac{\hat{q}(1-\hat{q}) / n}{(1-\hat{q})^{2}(\log (1-\hat{q}))^{2}}
$$

so that the within study variance for the $i$-th study is provided as

$$
\sigma_{i}^{2}=\frac{m_{i}-y_{i}}{m_{i} y_{i}\left(\log y_{i} / m_{i}\right)^{2}}+\frac{x_{i}}{n_{i}\left(n_{i}-x_{i}\right)\left(\log \left(1-x_{i} / n_{i}\right)\right)^{2}} .
$$

We acknowledge that the above are estimates of the variances of the diagnostic accuracy estimates, but are used as if they were the true variances.

Some important cases. If there are no further covariates, two important models are easily identified as special cases of (7). One is the fixed effects model

$$
\log \hat{\theta}_{i}=\beta_{0}+\epsilon_{i}
$$

and the other is the random effects model

$$
\log \hat{\theta}_{i}=\beta_{0}+\delta_{i}+\epsilon_{i}
$$

which have gained some popularity in the meta-analytic literature.

\section{Results}

\section{Case study on MMSE and dementia}

We illustrate the approach with an example and revisit a meta-analysis by Mitchell [26] on the diagnostic accuracy of the mini-mental state examination (MMSE) as a diagnostic test for the detection of dementia and, more recently, mild cognitive impairment (MCI). In this metaanalysis 38 studies were included and the entire data are reproduced in Table 2. We are interested in the question: is there a difference in diagnostic accuracy of the MMSE in the detection of dementia and MCI, as Figure 7 suggests.

We use proc mixed from the SAS software, version 9.2 for Windows [27], for the analysis (see also Table 3). 
Table 2 Meta-analysis of the diagnostic accuracy of the mini-mental state examination (MMSE) and dementia or mild cognitive impairment $(\mathrm{MCl})$ as reference standard; $\mathrm{TP}=$ true positives, $\mathrm{FN}=$ false negatives, $\mathrm{FP}=$ false positives, $\mathrm{TN}=$ true negatives

\begin{tabular}{|c|c|c|c|c|c|}
\hline Study & Condition & TP & $\mathrm{FN}$ & FP & $\mathrm{TN}$ \\
\hline 1 & Dementia & 65 & 3 & 240 & 870 \\
\hline 2 & Dementia & 117 & 12 & 10 & 110 \\
\hline 3 & Dementia & 48 & 19 & 63 & 989 \\
\hline 4 & Dementia & 134 & 8 & 28 & 152 \\
\hline 5 & Dementia & 24 & 5 & 44 & 292 \\
\hline 6 & Dementia & 67 & 15 & 48 & 153 \\
\hline 7 & Dementia & 64 & 17 & 1 & 71 \\
\hline 8 & Dementia & 281 & 64 & 20 & 286 \\
\hline 9 & Dementia & 13 & 1 & 44 & 286 \\
\hline 10 & Dementia & 262 & 20 & 29 & 177 \\
\hline 11 & Dementia & 143 & 18 & 29 & 123 \\
\hline 12 & Dementia & 183 & 33 & 33 & 51 \\
\hline 13 & Dementia & 22 & 1 & 152 & 140 \\
\hline 14 & Dementia & 112 & 1 & 590 & 2091 \\
\hline 15 & Dementia & 152 & 81 & 126 & 1009 \\
\hline 16 & Dementia & 29 & 26 & 26 & 236 \\
\hline 17 & Dementia & 31 & 6 & 3 & 247 \\
\hline 18 & Dementia & 10 & 3 & 12 & 333 \\
\hline 19 & Dementia & 707 & 88 & 1438 & 10447 \\
\hline 20 & Dementia & 181 & 108 & 17 & 184 \\
\hline 21 & Dementia & 59 & 29 & 23 & 74 \\
\hline 22 & Dementia & 74 & 23 & 16 & 143 \\
\hline 23 & Dementia & 27 & 12 & 26 & 209 \\
\hline 24 & Dementia & 40 & 6 & 75 & 528 \\
\hline 25 & Dementia & 317 & 52 & 173 & 578 \\
\hline 26 & Dementia & 387 & 116 & 16 & 54 \\
\hline 27 & Dementia & 118 & 65 & 1 & 44 \\
\hline 28 & Dementia & 44 & 7 & 34 & 396 \\
\hline 29 & Dementia & 123 & 46 & 98 & 309 \\
\hline 30 & Dementia & 25 & 43 & 3 & 171 \\
\hline 31 & Dementia & 73 & 32 & 2 & 225 \\
\hline 32 & Dementia & 37 & 45 & 1 & 440 \\
\hline 33 & Dementia & 78 & 34 & 45 & 376 \\
\hline 34 & $\mathrm{MCl}$ & 72 & 12 & 53 & 214 \\
\hline 35 & $\mathrm{MCl}$ & 106 & 23 & 410 & 379 \\
\hline 36 & $\mathrm{MCl}$ & 37 & 36 & 22 & 118 \\
\hline 37 & $\mathrm{MCl}$ & 67 & 30 & 22 & 75 \\
\hline 38 & $\mathrm{MCl}$ & 17 & 77 & 1 & 90 \\
\hline
\end{tabular}

The values of the dependent variable $\log \hat{\theta}_{i}$ are easily constructed from Table 2. We are interested to see if there are differences in accuracy for diagnosing MCI compared

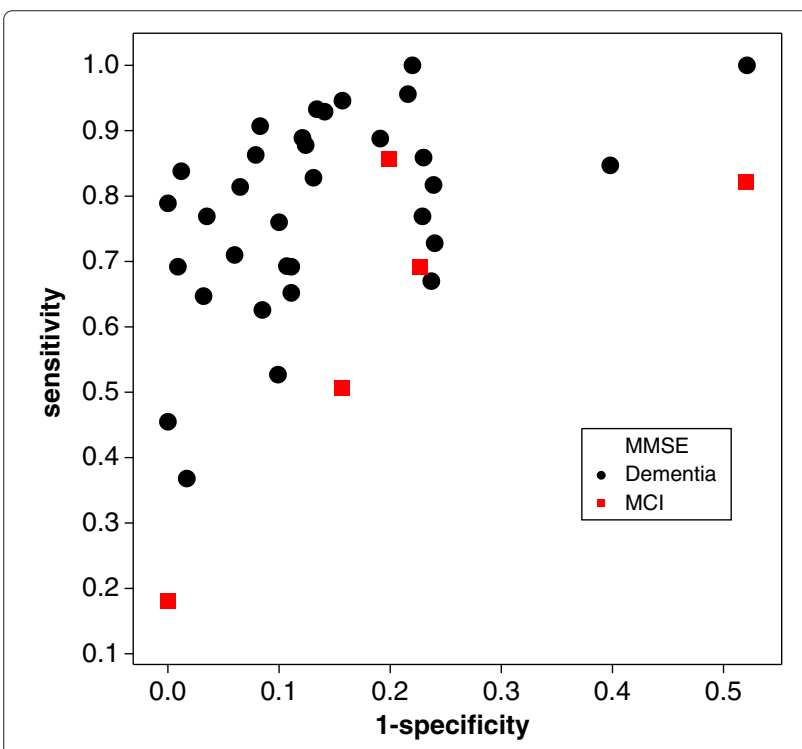

Figure 7 SROC diagram for the meta-analysis of MMSE and dementia or $\mathrm{MCl}$ as reference standard.

to diagnosing dementia. Hence we have constructed a covariate condition which takes the value 1 if the study concerns $\mathrm{MCI}$ as condition and 0 if the study is on dementia. Since we have fixed within-study variances, we need to tell proc mixed to incorporate this appropriately; this can be accomplished by using a weight, $w_{i}=$ $1 / \sigma_{i}^{2}$. The random option induces a random effect (here study) with associated variance component $\tau^{2}$, which is estimated. However, SAS proc mixed will automatically fit a within-study variance component (on top of the provided variances). To circumvent this mechanism, the option parms (1) (1) / hold=2 is used where the term hold=2 fixes the second variance component, corresponding to the within-study variance multiplier, to one. Note that the random effect modelling betweenstudy variation is described by a free variance parameter, $\tau^{2}$. For this a starting value needs to be given: we have $\tau^{2}=1$, although other choices are possible, e.g. $\tau^{2}=0$, corresponding to the case of no heterogeneity between studies.

The results of the analysis are provided in Table 4 . It can be seen that there is a significant effect of condition (dementia/MCI) on the diagnostic accuracy, with diagnostic accuracy being significantly higher in studies with patients having dementia in comparison to the diagnostic accuracy in studies with patients having mild cognitive impairment. Nevertheless, not all heterogeneity is explained by this covariate as the random effect (study effect) still remains significant, as the bottom part of Table 4 shows.

The inference is based here on a procedure called the Wald test. The estimated parameter value is divided by its 
Table 3 SAS proc mixed adapted for meta-analysis of diagnostic accuracy study data

\begin{tabular}{ll}
\hline SAS statement & Explanation \\
\hline proc mixed data=MMSE method=ml covtest ; & procedure mixed of SAS, data contains the data file, method \\
& specifies estimation \\
defines the categorical variables used & defines the model: LHS outcome, RHS covariates used \\
model logtheta $=$ condition/s; & w contains inverse variance as weight \\
weight w; & factor study nested in condition \\
random study (condition); & specifies starting values, hold=2 fixes the residual variance component \\
parms (1) (1)/hold=2; & executes the program \\
run; &
\end{tabular}

estimated standard error, and the result is given in column four in Table 4. The likelihood ratio test may be considered as an alternative. It is defined as two times the difference of the log-likelihood including the effect of interest and the log-likelihood not including the effect of interest. For the effect of condition in Table 4, we find a value of 6.8 for the likelihood-ratio test. The Wald test is asymptotically standard normal under the null-hypothesis of absence of effect, whereas the likelihood ratio test statistic is asymptotically chi-squared distributed with degrees of freedom equal to the number of parameters associated with the effect considered (in this case one). It is well-known that the likelihood ratio test is more powerful. Here, both tests provide similar p-values, with 0.0091 for the likelihood ratio test and 0.0069 for the Wald test; this confirms the significance of the effect (dementia/MCI) on the diagnostic accuracy.

It is trivial to construct the associated SROC curves from Table 4. We find

$$
\begin{aligned}
\text { for dementia: } p & =(1-q)^{\exp (-2.2878)}, \\
\text { for MCI: } p & =(1-q)^{\exp (-2.2878+0.8605)} \text {. }
\end{aligned}
$$

Note that the likelihood ratio test as well as the Wald test need modification in situations where the null hypothe-

\begin{tabular}{|c|c|c|c|}
\hline Effect & $\begin{array}{c}\text { Parameter } \\
\text { estimate }\end{array}$ & SE & Z-value \\
\hline \multicolumn{4}{|l|}{ fixed } \\
\hline Intercept & -2.2878 & 0.1208 & -18.94 \\
\hline condition & 0.8605 & 0.3187 & 2.70 \\
\hline \multicolumn{4}{|l|}{ random } \\
\hline$\tau^{2}($ study) & 0.3078 & 0.1049 & 2.90 \\
\hline
\end{tabular}
sis is part of the boundary of the alternative such as when testing $\mathrm{H}_{0}: \tau^{2}=0$. In this case, the asymptotic null distribution of the likelihood ratio test statistic is no longer $\chi^{2}$ with $1 \mathrm{df}$ but rather a mixture of a two-mass distribution giving equal weights 0.5 to the one-point mass distribution at 0 and a $\chi^{2}$ with $1 \mathrm{df}$ [28]. Practically, this means that standard 2-sided p-values have to be divided by 2 .

\section{Case study on MOOD and depressive disorders}

The MOOD module of the Patient Health Questionnaire (PHQ-9) has been developed to screen and to diagnose patients in primary care with depressive disorders. The instrument consists of 9 questions, each scored from 0 to 3 points with a total score ranging from 0 to 27 . In a meta-analysis of the diagnostic accuracy of MOOD, Wittkampf et al. [29] included 12 studies. These studies used either a cut-off of 10 (referred to here as "summary score") or a more complex evaluation algorithm ("algorithm"). The complete data are listed in Table 5 and the associated SROC diagram is given in Figure 8. The impression from the graph is that the cut-off of 10 used by

Table 5 Meta-Analysis of the diagnostic accuracy of the MOOD module and depression in patients in primary care as reference standard; TP = true positives, $F N=$ false negatives, $\mathrm{FP}=$ false positives, $\mathrm{TN}=$ true negatives

\begin{tabular}{llcccc}
\hline Study & Cut-off & TP & FN & FP & TN \\
\hline 1 & algorithm & 65 & 26 & 104 & 1192 \\
2 & algorithm & 70 & 13 & 74 & 846 \\
3 & sum score & 62 & 10 & 27 & 429 \\
4 & sum score & 36 & 5 & 65 & 474 \\
5 & sum score & 55 & 11 & 43 & 392 \\
6 & algorithm & 6 & 8 & 12 & 144 \\
7 & sum score & 121 & 103 & 80 & 720 \\
8 & algorithm & 11 & 5 & 5 & 76 \\
9 & algorithm & 6 & 5 & 0 & 3 \\
10 & algorithm & 85 & 31 & 9 & 460 \\
11 & sum score & 15 & 1 & 4 & 42 \\
12 & sum score & 96 & 10 & 23 & 187 \\
\hline
\end{tabular}




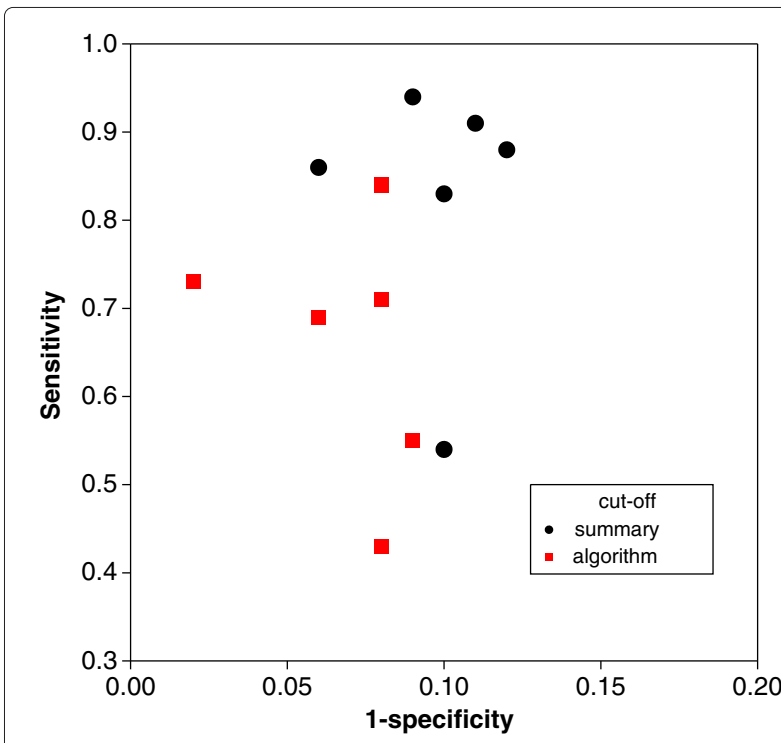

Figure 8 SROC diagram for the meta-analysis of MOOD and depression in patients in primary care.

the summary score has a higher diagnostic accuracy than the alternative.

The presence or absence of a cut-off value effect is now more formally investigated using a covariate cut-off, which is zero when the summary score with a cut-off value of 10 is used and one otherwise. The results are presented in Table 6. It can be seen that the covariate cut-off level "summary score" is associated with a higher diagnostic accuracy, although, as seen from the Wald statistics provided in column four of Table 6, the effect is not significant. We see a significant random effect (study; adjusted p-value 0.0274; see comment at the end of section "Case study on MMSE and dementia"), which indicates that the random study effect is needed in the analysis. It is not really surprising that the covariate cut-off is not significant, since the concept of the SROC is designed to accommodate the cut-off value variation. We will take up this point in the next section.

Table 6 Analysis of the cut-off effect for the meta-analysis of the MOOD module and depression in patients in primary care

\begin{tabular}{lccc}
\hline Effect & $\begin{array}{c}\text { Parameter } \\
\text { estimate }\end{array}$ & SE & Z-value \\
\hline fixed & & & \\
Intercept & -2.5332 & 0.2817 & -8.99 \\
cut-off & 0.4804 & 0.3966 & 1.21 \\
\hline random & & & \\
$\tau^{2}$ (study) & 0.3239 & 0.1690 & 1.92 \\
\hline
\end{tabular}

\section{Discussion}

\section{Global versus local criteria}

We have focussed on the $\mathrm{PH}$ measure so far, as it provides an appropriate measure for comparing SROC curves globally, in the sense that cut-off value variation will not necessarily effect the estimate of the SROC curve. The situation is illustrated in Figure 9.

Evidently, different cut-off values are associated with the same value of $\log \theta$, hence, the $\mathrm{PH}$ measure $\log \theta$ is not the best measure to discriminate different cut-off values. This is not surprising, since the SROC curve is a concept designed for assessing the diagnostic accuracy of a diagnostic test globally, in the sense that it adjusts for different cut-off values. Hence, a measure that assesses local performance of the diagnostic is needed. Assuming that every cut-off value used in the meta-analysis is clinically meaningful, we suggest use of the (squared) Euclidean distance to the upper left corner $(0,1)$ of the ROC diagram as a more meaningful measure to compare cut-off values:

$$
\hat{E}_{i}=\left(1-\hat{p}_{i}\right)^{2}+\left(1-\hat{q}_{i}\right)^{2},
$$

where $\hat{p}_{i}=y_{i} / m_{i}$ and $\hat{q}_{i}=x_{i} / n_{i}$. Each point in the SROC diagram has a unique circle with center $(0,1)$ that passes through this point. In Figure 9, one such circle is illustrated which also has the smallest Euclidean distance among the three available points (since it has smallest radius among the three possible points with associated circles). In the following, we will consider the criterion (14) as an alternative criterion to choose the cut-off value.

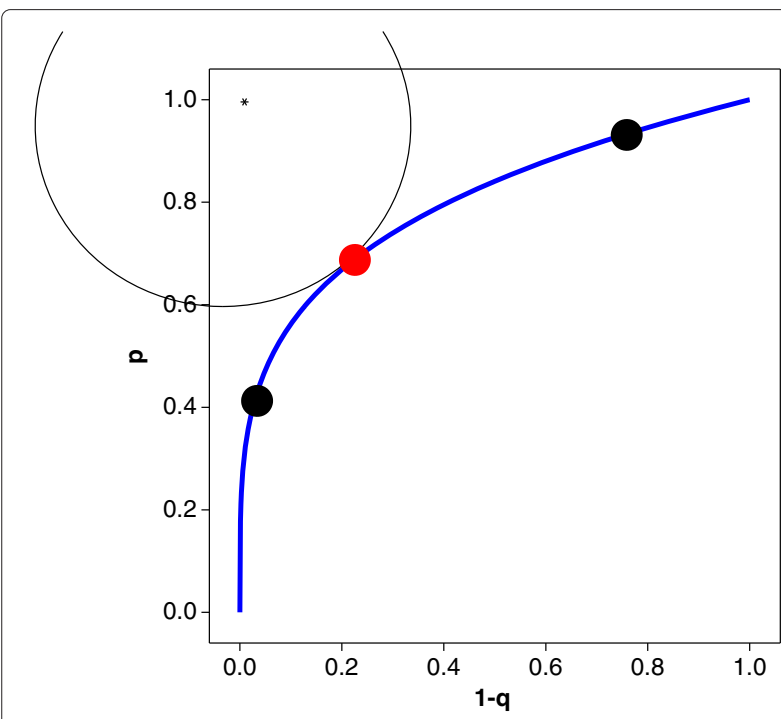

Figure 9 Different cut-off values with associated sensitivities and specificities on the same SROC curve with different Euclidean distances; the point on the circle has shortest Euclidean distance to the upper left vertex of the SROC diagram as indicated by the circle. 
Since we have changed the criterion, we need to determine the associated within-study variances. This can be accomplished easily, using the $\delta$-method once more, to obtain

$$
\operatorname{Var}(\hat{E}) \approx 4(1-\hat{p})^{2} \hat{p}(1-\hat{p}) / m+4(1-\hat{q})^{2} \hat{q}(1-\hat{q}) / n,
$$

where we have ignored study indexes for the the sake of simplicity. Using this criterion, we see in Figure 9 that cut-off values can vary considerably in their diagnostic accuracy, despite having identical diagnostic accuracy at a global level. We re-analyze the metaanalysis of MOOD and depression with respect to the (squared) Euclidean distance and provide the results in Table 7.

Evidently, both criteria lead to the same conclusion, namely that using the summary score with a cut-off value of 10 leads to the higher diagnostic accuracy (although the effect is not significant). It might also be worthwhile looking at the results of the likelihood ratio test: for the $\mathrm{PH}$-measure as the outcome variable, the likelihood ratio test provides a value of 1.5; for the Euclidean distance, the value of the likelihood ratio test is 1.7 , confirming the nonsignificance of the effect. Nevertheless, the analysis shows that the cut-off value of 10 provides the higher diagnsotic accuracy.

\section{Meta-analysis of magnetic resonance spectroscopy and prostate cancer.}

This case study provides an example where the use of a global or local criterion leads to a different conclusion. Magnetic resonance spectroscopy has the ability to discriminate between prostate cancer and benign prostatic hyperplasia, based on reduced citrate and elevated choline in the cancerous region. The diagnostic test works on a voxel of signal intensity ratios of (choline+creatine)/citrate. Two cut-off points are in use: $<0.75$ and $<0.86$. The results collected in a meta-analysis by Wang et al. [30] include 12 studies, as presented in Table 8; the associated SROC diagram is presented in Figure 10. From the graph, there is no obvious choice for the "best" cut-off value.

The fixed effects parts of the mixed model analysis, using the global PH measure and the local Euclidean measure as criteria, are presented in Table 9. It is interesting to note that the focus of the analysis, global or local,

Table 7 Analysis of the cut-off effect for the meta-analysis of the MOOD module and depression in patients in primary care

\begin{tabular}{llccc}
\hline Criterion & $\begin{array}{l}\text { Effect } \\
\text { estimate }\end{array}$ & Parameter & SE & Z-value \\
\hline PH measure & cut-off & 0.4804 & 0.3966 & 1.21 \\
Euclidean distance & cut-off & 0.0563 & 0.0430 & 1.31 \\
\hline
\end{tabular}

Table 8 Meta-analysis of the magnetic resonance spectroscopy and prostate cancer; TP = true positives, $\mathrm{FN}=$ false negatives, $\mathrm{FP}=$ false positives, $\mathrm{TN}=$ true negatives

\begin{tabular}{lccccc}
\hline Study & Cut-off & TP & FN & FP & TN \\
\hline 1 & 0.75 & 122 & 30 & 35 & 55 \\
2 & 0.75 & 73 & 8 & 80 & 219 \\
3 & 0.75 & 75 & 6 & 92 & 207 \\
4 & 0.75 & 123 & 39 & 38 & 50 \\
5 & 0.75 & 134 & 21 & 40 & 39 \\
6 & 0.75 & 12 & 12 & 7 & 75 \\
\hline 7 & 0.86 & 81 & 71 & 24 & 59 \\
8 & 0.86 & 56 & 25 & 32 & 267 \\
9 & 0.86 & 52 & 29 & 20 & 59 \\
10 & 0.86 & 98 & 57 & 20 & 59 \\
11 & 0.86 & 6 & 9 & 15 & 266 \\
12 & 0.86 & 44 & 8 & 32 & 264 \\
\hline
\end{tabular}

is an important part of the analysis. Globally, the better diagnostic accuracy is given by the cut-off value of 0.75 , whereas better local performance is achieved with a cutoff value of 0.86 , although neither analysis is significant.

\section{PH measure and positive likelihood ratio}

Another frequently used diagnostic measure is the positive likelihood ratio, defined as the ratio of sensitivity to false positive rate $P(T=1 \mid D=1) / P(T=1 \mid D=0)$ or $p /(1-q)$. It is different to the $\mathrm{PH}$ measure in that

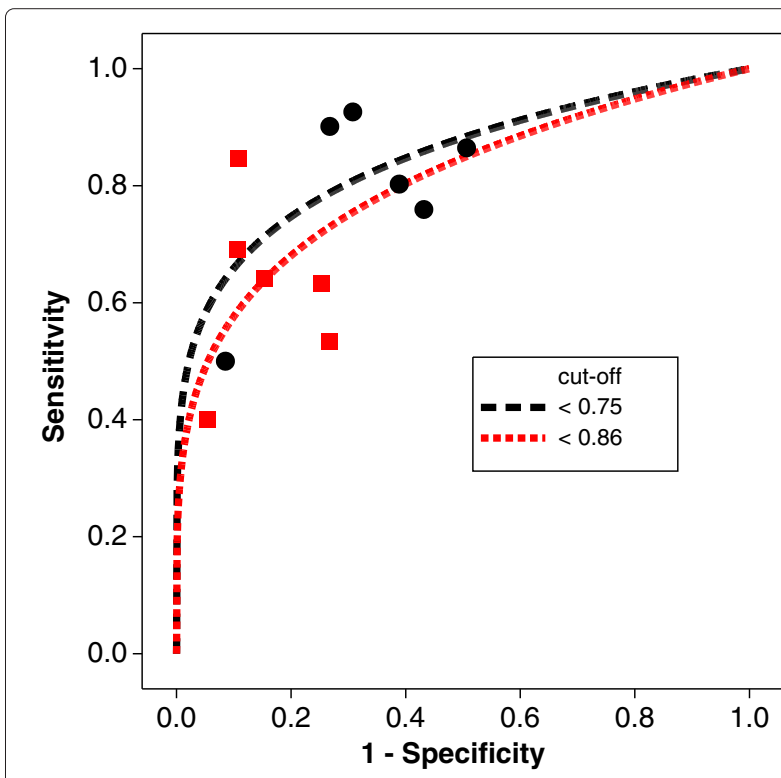

Figure 10 SROC diagram for the meta-analysis of the magnetic resonance spectroscopy and prostate cancer. 
Table 9 Analysis of the cut-off effect for the meta-analysis of the magnetic resonance spectroscopy and prostate cancer

\begin{tabular}{llccc}
\hline Criterion & Effect (reference) & $\begin{array}{c}\text { Parameter } \\
\text { estimate }\end{array}$ & SE & Z-value \\
\hline PH measure & cut-off $(<0.75)$ & 0.2049 & 0.3516 & 0.58 \\
Euclidean distance & cut-off $(<0.75)$ & -0.0212 & 0.0573 & -0.37 \\
\hline
\end{tabular}

the ratio is taken on the $\log$-scale: $\theta=\log p / \log (1-q)$. Furthermore, if re-expressed as models, the positivelikelihood ratio corresponds to $p=\theta^{\prime}(1-q)$, a straight line with no intercept, whereas the the $\mathrm{PH}$ measure corresponds to $p=(1-q)^{\theta}$, a straight line on the log-scale with no intercept. The positive likelihood ratio is a natural measure since it transfers the concept of relative risk (risk of a positive test in the diseased group to the risk of a positive test in the non-diseased group) to the diagnostic setting. However, it is less suitable as an (S)ROC model since it does not provide a function which connects the lower left vertex with the upper right vertex in the ROC diagram (which, in contrast, the $\mathrm{PH}$-model does provide).

\section{Conclusions}

The approach presented here is attractive since it is based on a simple measure of diagnostic accuracy per study, namely the ratio of $\log$-sensitivity to $\log$-false-positive rate. It also embeds the diagnostic meta-analysis problem into the well-known and much used mixed model setting. In particular, the analysis of effects of observed covariates on the diagnostic accuracy can easily be incorporated.

Controversies in the meta-analysis of diagnostic studies usually focus on comparability of studies. Study types might be case-control, cohort, cross-sectional or other. Studies might differ in the gold standard, severity of disease, or in the application of the diagnostic test. Patient populations might differ across studies, as might the cutoff value (defining positivity of the diagnostic test). All these different aspects, if observed, can be easily incorporated and analyzed as fixed effects in the special mixed model suggested here.

The occurrence of heterogeneity in the meta-analysis of diagnostic studies is more the rule than the exception; it is thus important to quantify the heterogeneity across studies due to the different sources. The approach provided here offers a more detailed investigation of heterogeneity according to the various observed sources and a residual heterogeneity (measured by $\tau^{2}$ ). This might allow us to construct a measure of relative residual heterogeneity, which might help to assess how trustworthy the results of a given meta-analysis may be. This will be investigated in future research.

In a recent study on the meta-analytical evaluation of coronary CT angiography studies, Schuetz et al. [31] investigated the problem of non-evaluable results that occur in the individual studies. They conclude that diagnostic accuracy measures change considerably depending on how non-evaluable results are treated. In fact, they conclude that

parameters for diagnostic performance significantly decrease if non-evaluable results are included by a $3 \times 2$ table for analysis (intention to diagnose approach).

Twenty-six studies were included in their meta-analysis with a wide range of non-evaluable results from 0 to 43 . Using the approach suggested here, it would be very easy to analyze the effect of non-evaluable results on the diagnostic accuracy by including the amount of non-evaluable results per study as a fixed effect in the proposed mixed model.

Competing interests

The authors declare that they have no competing interests.

\section{Authors' contributions}

SC carried out all statistical and computing analysis. WB collected and prepared all meta-analytic data sets. $\mathrm{HH}$ conceived the theoretical modelling work and DB drafted the idea of the approach and finalized the manuscript. All authors read and approved the final manuscript.

\section{Acknowledgements}

This work was funded by the German Research Foundation (GZ: Ho1286/7-2). We are very grateful to two referees for their insightful, inspiring and helpful comments. Thanks go also to Dr Sean Ewings for a careful and critically reading of the manuscript.

\section{Author details}

${ }^{1}$ Health Sciences Program Udon Thani Rajabhat University, Udon Thani, Thailand. ${ }^{2}$ Statistics and Quantitative Methods, Faculty of Psychology and Sport Science, University of Münster, Münster, Germany. ${ }^{3}$ Southampton Statistical Sciences Research Institute, Mathematics and Medical Statistics, University of Southampton, Southampton SO17 1BJ, UK.

Received: 11 October 2013 Accepted: 14 April 2014

Published: 24 April 2014

\section{References}

1. Hasselblad V, Hedges LV: Meta-analysis of screening and diagnostic tests. Psychol Bull 1995, 117:167-178.

2. Sutton AJ, Abrams KR, Jones DR, Sheldon TA, Song F: Methods for Meta-Analysis in Medical Research. New York: Wiley; 2000.

3. Deeks JJ: Systematic reviews of evaluations of diagnostic and screening tests. In Systematic Reviews in Health Care: Meta-Analysis in context, vol.14, pp.248-282. London: BMJ Books; 2007.

4. Schulze R, Holling H, Böhning D: Meta-Analysis. New Developments and Applications in Medical and Social Sciences, Hogrefe \& Huber: Göttingen; 2003.

5. Pepe MS: Receiver operating characteristic methodology. J Am Stat Assoc 2000, 95:308-311.

6. Pepe MS: The Statistical Evaluation of Medical Tests for Classification and Prediction. Oxford: Oxford University Press; 2003.

7. Holling H, Böhning W, Böhning D: Meta-analysis of diagnostic studies based upon SROC-curves: a mixed model approach using the Lehmann family. Stat Modelling - Int J 2012, 12:347-375.

8. Swets JA: Signal Detection Theory and ROC Analysis in Psychology and Diagnostics Collected Papers. New York, London: Psychology Press Taylor \& Francis Group; 1996.

9. Doust JA, Glasziou PP, Pietrzak E, Dobson AJ: A system reviews of diagnostic accuracy of natriyretic peptides for heart failure. Arch Intern Med 2004, 2:9. 
10. Youden D: Index for rating diagnostic tests. Cancer 1950, 3:32-35.

11. Glas AS, Lijmer JG, Prins MH, Bonsel GJ, Bossuyt PMM: The diagnostic odds ratio: a single indicator of test performance. J Clin Epidemiol 2003, 56:1129-1135.

12. Moses $L E$, Shapiro $D$, Littenberg B: Combining independent studies of a diagnostic test into a summary ROC curve: data-analytic approaches and some additional considerations. Stat Med 1993, 12:1293-316.

13. Littenberg B, Moses LE: Estimating diagnostic accuracy from multiple conflicting reports: A new meta-analytic method. Med Decis Making 1993, 13:313-321.

14. Rutter $C M$, Gatsonis $C A$ : $\mathbf{A}$ hierarchical regression approach to meta-analysis of diagnostic test accuracy evaluations. Stat Med 2001, 20:2865-2884.

15. Walter SD, Macaskill P: SROC curve. In Encyclopedia of Biopharmaceutical statistics. New York: Marcel Dekker; 2004

16. Van Houwelingen $\mathrm{HC}$, Zwinderman $\mathrm{KH}$, Stijnen T: A bivariate approach to meta-analysis. Stat Med 1993, 12:2273-2284.

17. Reitsma JB, Glas AS, Rutjes AWS, Scholten RJPM, Bossuyt PM, Zwinderman $\mathrm{AH}$ : Bivariate analysis of sensitivity and specificity produces informative measures in diagnostic reviews. $J$ Clin Epidemiol 2005, 58:982-990.

18. Arends $L R$, Hamza $T H$, van Houwelingen JC, Heijenbrok-Kal MH, Hunink MGM, Stijnen T: Bivariate random effects meta-analysis of ROC curves. Med Decis Making 2008, 28:621-638.

19. Harbord RM, Deeks JJ, Egger M, Whiting P, Sterne JAC: A unification of models for meta-analysis of diagnostic accuracy studies. Biostatistics 2006, 1:1-21.

20. Liu X: Classification accuracy and cut point. Stat Med 2012, 31:2676-2686.

21. Le CT: $\mathbf{A}$ solution for the most basic optimization problem associated with an ROC curve. Stat Methods Med Res 2006, 15:571-584

22. Gönen M, Heller G: Lehmann family of ROC curves. Med Decis Making 2010, 30:509-517.

23. Aertgeerts FB, Kester A: The value of the CAGE in screening for alcohol abuse and alcohol dependence in general clinical populations: a diagnostic meta-analysis. J Clin Epidemiol 2003, 57:30-39.

24. Rücker G, Schumacher M: Letter to the editor. Biostatistics 2009, 10:806-807.

25. Rücker G, Schumacher M: Summary ROC curve based on a weighted Youden index for selecting an optimal cutpoint in meta-analysis of diagnostic accuracy. Stat Med 2010, 29:3069-3078.

26. Mitchell AJ: A Meta-Analysis of the accuracy of the mini-mental state examination in the detection of dementia and mild cognitive impairment. J Psychiatr Res 2009, 43:411-431.

27. SAS Institute Inc: SAS/STAT(R) 9.2 User's Guide, Second Edition. Cary, NC, USA: SAS Insitute Inc.; 2008

28. Self SG, Liang K-Y: Asymptotic properties of maximum likelihood estimators and likelihood ratio tests under nonstandard conditions. J Am Stat Assoc 1987, 82:605-610.

29. Wittkampf KA, Naeije L, Schene AH, Huyser J, van Weet HC: Diagnostic accuracy of the mood module of the patient health questionnaire: a systematic review. Gen Hosp Psychiatry 2007, 29:388-395.

30. Wang P, Guo YM, Qiang YQ, Duan XY, Zhang QJ, Liang W: A meta-analysis of the accuracy of prostate cancer studies which use magnetic resonance spectroscopy (MRS) as a diagnostic tool. Korean J Radiol 2008, 9:432-438.

31. Schuetz GM, Schlattmann P, Dewey M: Use of 3CE2 tables with an intention to diagnose approach to assess clinical performance of diagnostic tests: meta-analytical evaluation of coronary CT angiography studies. $B M J$ 2012, 345:6717.

doi:10.1186/1471-2288-14-56

Cite this article as: Charoensawat et al:: Meta-analysis and meta-modelling for diagnostic problems. BMC Medical Research Methodology 2014 14:56.

\section{Submit your next manuscript to BioMed Central} and take full advantage of:

- Convenient online submission

- Thorough peer review

- No space constraints or color figure charges

- Immediate publication on acceptance

- Inclusion in PubMed, CAS, Scopus and Google Scholar

- Research which is freely available for redistribution
C Biomed Central 